\title{
Directional control of dissociative ionization by a two-colour laser field
}

\author{
Vincent Wanie $^{1,2}$, Heide Ibrahim ${ }^{1, *}$, Samuel Beaulieu ${ }^{1}$, Nicolas Thiré ${ }^{1}$, Bruno E. Schmidt ${ }^{1,3}$, \\ Yunpei Deng ${ }^{4}$, Ali S. Alnaser ${ }^{5}$, Igor V. Litvinyuk ${ }^{6}$, Xiao-Min Tong ${ }^{7}$, and François Légaré ${ }^{1}$ \\ ${ }^{1}$ Institut National de la Recherche Scientifique, Varennes, QC, J3X 1S2, Canada \\ ${ }^{2}$ Institute for Photonics and Nanotechnologies CNR-IFN,20133 Milano, Italy \\ ${ }^{3}$ few-cycle Inc., Montreal, Qc, H1L 5W5, Canada \\ ${ }^{4}$ Paul Scherrer Institut, CH-5232 Villigen, Switzerland \\ ${ }^{5}$ Physics Department, American University of Sharjah, Sharjah, POB26666, UAE \\ ${ }^{6}$ Centre for Quantum Dynamics, Griffith University, Queensland 4111, Australia \\ ${ }^{7}$ Center for Computational Sciences and Faculty of Pure and Applied Sciences, University of \\ Tsukuba, Ibaraki, 305-8577 Japan
}

\begin{abstract}
Using asymmetric two-color laser fields composed of 1800 and $900 \mathrm{~nm}$, we have simultaneously controlled four well identified fragmentation channels in dissociative ionization of the hydrogen molecule, resulting in enhanced electron-localization sensitivities of up to $65 \%$.
\end{abstract}

\section{Control principle}

The interaction of an asymmetric laser field with a molecular system can lead to efficient control in the directionality of its dissociative ionization. Whether an electron remains on one side or the other of a dissociating molecule depends on the composition of this electric field; due to the longer period of one optical cycle (6.0fs at $1800 \mathrm{~nm}$ versus $2.7 \mathrm{fs}$ at $800 \mathrm{~nm}$ ), the mid-infrared (MIR) spectral range enables a more efficient subcycle control of slower nuclear dynamics compared to $800 \mathrm{~nm}$, which are the preponderant dynamics in large molecular systems. Also for the simplest of all molecules, the hydrogen cation, one can achieve excellent timing for the coupling of the electronic states involved, $\sigma_{\mathrm{g}}$ and $\sigma_{\mathrm{u}}$ (see Fig. 1a), since half a vibrational period matches the optical cycle. This leads to very efficient control of electron localization. At the same time, MIR photons comprise a smaller energy (0.69eV@1800nm vs. 1.55eV @ 800nm), which opens up additional interference pathways when overcoming the energy gap of relevant electronic states, due to more photon-combination possibilities.

This variety further enriches the "play on the control-piano" of dissociation channels.

\footnotetext{
*Corresponding author: ibrahim@emt.inrs.ca
} 


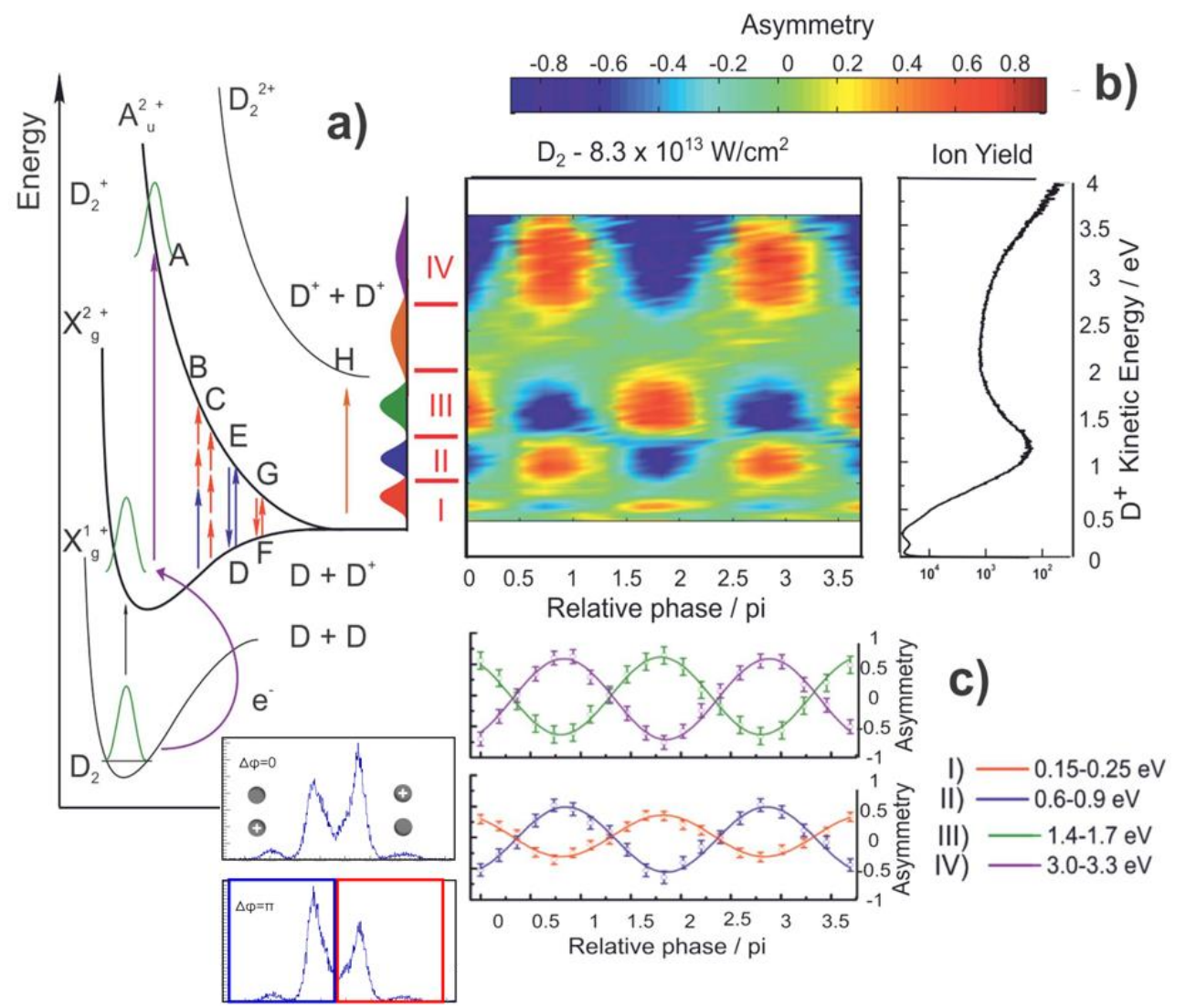

Fig. 1. Principle of $\mathrm{D}_{2}{ }^{+}$dissociation control experiment with control channels at different kinetic energy (KE) regions, as indicated with roman numbers. (a) Scheme of the reflection principle associated to the formation of $\mathrm{D}^{+}$ions from bond softening (red $\mathrm{KE}$ region), above-threshold dissociation (blue KE region), three-photon dissociation (green KE region), charge-resonanceenhanced ionization (orange KE region, no control) and recollision excitation (purple KE region). Capital letters correspond to different pathways. Blue arrows indicate absorption of $2 \omega$ photons, red arrows of $\omega$ photons. The two insets show the occurring asymmetry depending on the relative phase $\varphi$ in the time-of-flight spectrum. The left two peaks correspond to $\mathrm{D}^{+}$fragments pointing towards the detector, the right two peaks correspond to $\mathrm{D}^{+}$fragments pointing away from it (b) left panel: experimental calculated asymmetries for the detected $\mathrm{D}^{+}$fragments at an intensity of $8.3 \times 10^{13}$ $\mathrm{Wcm}^{-2}$ and right panel: corresponding logarithmic energy distribution. (c) Averaged asymmetries for the specified KE cuts.

\section{Results}

For the experiments reported here, MIR two- colour (1800 and 900nm) laser pulses of 50fs duration were used to coherently control the dissociative ionization of $\mathrm{H}_{2}$ and $\mathrm{D}_{2}$ molecules [1,2]. In the two-colour technique, which is experimentally significantly easier to implement compared to CEP stabilized few-cycle pulses, an asymmetry over such a relatively long duration can be achieveds, since the electric field can basically be tailored on any chosen timescale. This is because in the case of CEP stabilization the time-averaged electric field goes to zero with increasing 
duration, in contrary to two- colour fields where an asymmetry is imprinted on every single cycle and thus remains independent on the pulse duration.

With this two-colour approach we have obtained control over four distinct dissociation pathways, occurring from different areas of kinetic energy (KE), as indicated in Fig.1, utilizing the normalized asymmetry parameter $A(\varphi, E)=\left(N_{u p}(\varphi, E)-N_{\text {down }}(\varphi, E)\right) /\left(N_{u p}(\varphi, E)+N_{\text {down }}(\varphi, E)\right)$ :

(I) Bond Softening channel (red and blue region of kinetic energy - KE, indicated in Fig. 1a): Absorption of one $\omega$ or one $2 \omega$ photon (pathways $G$ and $\mathrm{E}$, respectively) can lead to dissociation of the molecule via the $\sigma_{\mathrm{u}}$ cationic state (net one photon).

(II) Above threshold dissociation (ATD, blue KE range): E.g. three photon absorption (C) followed by stimulated emission of one photon (F) may lead to dissociation of the molecule via the $\sigma_{\mathrm{g}}$ cationic state (net two photons).

(III) Three-photon dissociation (green KE range): E.g. three photon absorption (C) (net three photons).

(IV) Recollision excitation (RE, purple KE region): While channels (I-III) arise from radiative coupling of the molecule with the laser field, channel (IV) results from an inelastic recollision of the ionized electron with its remaining molecular ion. This leads to an excitation of the molecule to the $\sigma_{u}$ dissociative state (A). Because the dissociative state is populated rather early, the generated ions possess a high KE.

The actual control effect occurs from an overlap of involved nuclear wavepackets in space and time on the potential energy surfaces - in general the case when their kinetic energy distribution is similar. This leads to tuneable interferences between dissociation pathways, each populating electronic states of different parity via the concurrent net absorption of $n$ and $n+1$ photon(s) in the presence of a strong laser field. As an example, because BS (pathway E) and ATD (pathways $\mathrm{C}+\mathrm{F}$ ) both lead to the same final KE but with wavepackets propagating on different electronic states $\left(\sigma_{\mathrm{g}}\right.$ and $\sigma_{\mathrm{u}}$ ), the resulting interference allows us to coherently control the dissociation channel of KE region II. Hereby enhanced electron-localization sensitivities of up to $65 \%$ are achieved.

The experimental results are well reproduced by a theoretical model solving the time-dependent Schrödinger equation for the molecular ion, identifying the involved dissociation mechanisms. Furthermore, the comparison between theory and experiment allowed us to highlight the importance of the initial vibrational distribution on electron localization. We have found that in our case fewer vibrational states are being involved, compared to a Franck Condon type distribution.

\section{References}

1. V. Wanie, H. Ibrahim, S. Beaulieu, N. Thiré, B. E. Schmidt, Y. Deng, A. S. Alnaser, I. V. Litvinyuk, X.-M. Tong, F. Légaré, J. Phys. B: At. Mol. Opt. Phys. 49, 025601 (2016)

2. H. Ibrahim, C. Lefebvre, A. D. Bandrauk, A. Staudte, F. Légaré, J. Phys. B: At. Mol. Opt. Phys., 51, 042002 (2018) 